\title{
Persistent pulmonary interstitial emphysema with respiratory infection: a clinicopathological analysis of six cases and detection of infectious pathogens by metagenomic next-generation sequencing (mNGS)
}

\author{
Ping Zhou ${ }^{1}$, Weiya Wang ${ }^{1}$, Qian $\mathrm{Wu}^{1}$, Yiyun $\mathrm{Fu}^{1}$, Ying Zhang ${ }^{1}$, Zuoyu Liang ${ }^{1}$, Yuan \\ Tang $^{1}$, and Lili Jiang ${ }^{2}$ \\ ${ }^{1}$ Sichuan University West China Second University Hospital Department of Pathology \\ ${ }^{2}$ Sichuan University West China Hospital
}

October 20, 2020

\begin{abstract}
Objective: Persistent pulmonary interstitial emphysema (PPIE) is always related to mechanical ventilation and preterm. Its relationship with respiratory infection has rarely been reported in the literature. PPIE is difficult to diagnosis, always mimics with other cystic lesions. The objective of this study was to evaluate clinicopathological radiographic features of PPIE with respiratory infection and to detect the possible infectious pathogens. Methods: We retrospectively reviewed a total of 237 patients pathologically diagnosed with cystic lesions in West China Hospital of Sichuan University from January 2011 to April 2019. This retrospective cohort study analyzed clinicopathological radiographic features and to detect the infectious pathogens by metagenomic next-generation sequencing (mNGS). Results: Six cases were presented with primary syndrome of respiratory infection. There were four girls and two boys, ranged from 2 months to 5 years. $100 \%(5 / 5)$ available cases were full-term and without mechanical ventilation. CCAM were suspected in $66.7 \%(4 / 6)$ patients. $66.7 \%(4 / 6)$ cases affected only a single lobe, and $33.3 \%(2 / 6)$ cases affected both lung lobes. The pathologic characteristics showed lung cysts with variable size along the bronchovaslcular bundles, the cysts had a discontinuous fibrotic wall with a smooth inner surface, lined with uninucleated and/or multinucleated macrophages. Conclusions: Six rare cases of PPIE with respiratory infection were treated by lobectomy. All available five cases were full-term infants without mechanical ventilation. And we firstly tried to detect of infectious pathogens by mNGS, however, there was no certain infectious pathogen associated with PPIE in our study.
\end{abstract}

\section{Introduction}

With routine prenatal ultrasound scans performed, more and more congenital cystic lesions of the lung are diagnosed in infants[1], but the incidence is rare, which is present in 1 per 10,000-35,000 births[2]. The differential diagnosis of congenital cystic lesions contains congenital cystic adenomatoid malformation (CCAM), pulmonary sequestration (PS), bronchogenic cyst, congenital lobar emphysema (CLE), and persistent pulmonary interstitial emphysema (PPIE) and so on.

Pulmonary interstitial emphysema (PIE) is a rare cystic disease of infants. PIE is an air leak syndrome, characterized by gas dissecting pulmonary interstitium, along the bronchovascular bundles. There are three clinical types of PIE, including acute IPE, local persistent PIE (LPPIE) and diffuse persistent PIE (DPPIE) $[3,4]$. Acute IPE is lesser than seven days in duration, diffuse persistent PIE is observed when small cysts are noted in all lobes of the lung, while local persistent PIE affected only one lobe [5]. Chest computed tomography (CT) scan sometimes was limited to diagnose PIE. Sometimes, CT showed cystic lung lesions mimicking CCAM in the postnatal period[6]. The definitive diagnosis is histological. A histological diagnosis 
of PIE was established through the wall of cysts composed of a thin layer of discontinuous fibrous tissue and lined with uninucleated or/and multinucleated macrophages $[7,8]$.

PIE frequently occurs in premature infants with positive pressure mechanical ventilation $[9,10]$. However, it has also been reported rarely in both nonventilated infants and full-term infants[11, 12]. Pursnani et al.[12] showed a 3-month-old infant with LPPIE who had no history of respiratory distress syndrome (RDS) and mechanical ventilation, the patient had a medical history of viral pneumonia 1 month prior to surgery, indicating respiratory infection may be associated with PPIE.

However, there were just a few reports of PPIE with respiratory infection[11-19], and possible infectious pathogens were still unclear. With the development of molecular methods of identification, the metagenomic next-generation sequencing (mNGS) is a novel, rapid, simple, and convenient approach in the clinical identification of infectious diseases.

In present study, we reported six rare cases of PPIE with respiratory infection, followed by successfully surgical lobectomy. To our best knowledge, it is the first time to detect the possible infectious pathogens in PPIE by using mNGS.

\section{Methods}

\subsection{Case series and clinicopathology features}

237 patients were pathologically diagnosis with cystic lesions from the pathology files in West China Hospital of Sichuan University from January 2011 to April 2019. All the original slides were reviewed and identified independently by two pathologists (P Zhou and LL Jiang). Clinical and radiographic features were obtained retrospectively from the patients' medical records and follow-up, including age, sex, term, mechanical ventilation, clinical features, CT diagnosis, affected sites and the diameter of the cystic lesions.

\subsection{Special stain}

Special stains (acid fast stain, Gomori's methenamine silver staining, and Giemsa) and TB-PCR (Qiagen) were carried out for all cases according to the manufacturer's protocol.

\subsection{DNA extraction, Library construction and sequencing}

DNA was extracted from available blocks with the TIANamp Micro DNA Kit (DP316, Tiangen Biotech) following the manufacturer's protocol. We constructed DNA libraries according to the standard protocol through end-repaired adapter added overnight, and by applying polymerase chain reaction amplification to the extracted DNA. To measure the adapters before sequencing, quality control was carried out using a bioanalyzer (Agilent 2100, Agilent Technologies, Santa Clara, CA, USA) combined with quantitative PCR. DNA sequencing was then performed with the BGISEQ-100 platform.

\subsection{Data processing and analysis}

High-quality sequencing data were generated after removing low quality, low complexity, and shorter reads. The data mapped to the human reference genome (hg19) were excluded using a powerful alignment tool called Burrows-Wheeler Alignment to eliminate the effect of the human sequences. The database used for the present study includes 6039 bacteria, 4945 viruses, 1064 fungi, which all related to human disease. Finally, the mapped data were processed after filtering out duplicate reads for advanced analysis. The SoapCoverage from the SOAP website was used to calculate the sequence depth and genomic coverage for each species.

\section{Results}

\subsection{Clinical characteristics}

Totally, 2.5\% (6/237) cases with cystic lesions were confirmed pathologically with PIE between January 2011 and April 2019 in the Department of pathology, West China Hospital of Sichuan University. And all the patients were treated with suggested therapy and then with surgery. 
The clinical characteristics of all the six patients are showed inTable 1 . There were four girls and two boys, ranged from 2 months to 5 years old. We collected the follow-up data of all patients except for No.4. No.4 patient had the wrong phone number. The other available patients $(5 / 5,100 \%)$ were all full-term without mechanical ventilation. The common symptoms of the patients were cough, fever and expectoration. All patients with PPIE were presenting as lung cysts with primary syndrome of respiratory infection. The cystic lesion was located at a single lobe among $66.7 \%$ (4/6) patients, who were identified with local PPIE. $33.3 \%$ $(2 / 6)$ patients affected both lung lobes, who were identified with diffuse PPIE.

Besides, patient No.5 has suffered the disease Langerhans cell histiocytosis (LCH) in the left submandibular lymph node for 1 year. She was asymptomatic when she has received the suggested chemotherapy treatment four times, she had suffered recurrent pneumonia for 3 months, and the routine examination of the chest CT found the diffuse cystic lesions in both lung lobes. In addition, she had the defect of ventricular septal defect.

\subsection{Imaging findings}

Prenatal ultrasound did not find any lesions in all six patients according to the medical records and followup. Chest X-ray showed translucency of the affected site (Fig. 1A). The CT scan of all the cases showed cystic lesions. According to the CT findings, CCAM was suspected in $66.7 \%$ (4/6) cases in present study. The radiographic patterns of PIPE ranged from a cystic lesion or multi-cystic lesion of one or more lobes to a diffuse multi-cystic involvement of all lobes. CT scan of the chest showed a single cyst (Fig. 1B) and bilateral cysts in both lungs (Fig. 1C). The affected site was the right lower lobe in $50 \%(3 / 6)$ patients, the right upper lobe in 16.7\% (1/6) patient. The local multi-cystic pattern in 66.7\% (4/6) LPPIE cases included a wide range of sizes for the cystic lesions (1.8 to $3.8 \mathrm{~cm})$. However, there are $33.3 \%(2 / 6)$ patients affected both lung lobes.

\subsection{Pathological examination}

All the patients were treated with surgery. The resected affected site was the right lower lobe in $50 \%(3 / 6)$, patients the right upper lobe in $16.7 \%(1 / 6)$ patient. However, there are $33.3 \%(2 / 6)$ patients (No.3 and No.5) affected both lung lobes, the resection of right lower lobe was made in patient No.3, and the resection of left lobe was made in patient No.5.

The gross specimens showed multiloculated cysts with variable size within the pulmonary parenchymal, and the cysts had a smooth inner surface. Some cysts contained a small amount of clear fluid.

Histological examination showed that the walls of the cysts were adjacent to interlobular septa or bronchovascular bundles, the wall of cysts was composed of a thin layer of fibrous tissue, and the thin band of fibrous tissue was discontinuous. Small collections of uninucleated and multinucleated macrophages were lined in the surface of the main cysts (Fig. 2 and Fig. 3). The giant cells contained from 2 to 40 centrally placed nuclears.

The adjacent parenchymal surrounded the cysts showed mild to marked atelectasis and inflammatory cells infiltrates in all cases(Fig. 2 and Fig. 3), containing histocytes, lymphocytes, plasma cells, and neutrophils, indicating there were inflammation along the cysts.

\subsection{Special stain and TB-PCR}

Special stains (acid fast stain, Gomori's methenamine silver staining, and Giemsa) and TB-PCR (Qiagen) were carried out for all cases according to the manufacturer's protocol. None was seen in the case series, indicating did not contain any identifiable organism or foreign material in all cases.

\subsection{Infectious pathogens detected by mNGS}

The next-generation sequencing was performed from blocks of resected lung samples for each patient. All the six blocks were available. In the current study, mNGS successfully identified the infectious pathogens in all patients, and the pathogens detected were showed in Table 2. Streptococcus pneumonia (specific reads: 
941) was detected in patient No.1, whose infectious symptoms were present when surgery. Patient No.5 has suffered the disease Langerhans cell histiocytosis $(\mathrm{LCH})$ in the left submandibular lymph node for 1 year, and received the suggested chemotherapy treatment four times, she had suffered recurrent pneumonia for 3 months. Neisseria mucosa (specific reads: 587), Neisseria sicca (specific reads: 247), Prevotel lamelaninogenica (specific reads: 258), and Prevotella histicola (specific reads: 174) and Fusobacterium nucleatum (specific reads: 239) were detected in case 5, which may be associated with the chemotherapy treatment for LCH. No infectious pathogen was detected in other 66.7\% (4/6) cases (patient No.2 to No.4, and No. 6), whose infectious symptoms were controlled with suggested therapy before surgery.

\section{Discussion}

The pathophysiology of pulmonary interstitial emphysema (PIE) is a result of air leakage into interstitium from alveolus due to disruption of the alveolar wall basement membrane, which may dissect along the bronchovascular bundles and radiates outwards to the periphery of the lung, mediastinum and pericardium. PIE included local persistant PIE and acute PIE, and diffuse persistent PIE[3, 4]. Diffuse persistent PIE is observed when small cysts are noted in all lobes of the lung[5], and acute IPE is lesser than 7 days in duration.

PIE is a rare condition that commonly affects newborn infants with a history of prematurity with positive pressure mechanical ventilation $[9,10]$. However, it has also been reported rarely in both nonventilated infants and full-term infants[11, 12]. There have been only a few cases reported for PIE developing in unventilated neonates $[3,11,12,18,20-22]$. In our study, five full-term unventilated infants underwent surgical lobectomy were diagnosed with the PPIE. However, all the patients were diagnosed with PPIE with respiratory infection.

Chest CT sometimes may find air surrounding the bronchovascular bundles in patients with PIE[3]. A Multi-institutional research found that about $82 \%$ patients with PPIE had the characteristic CT findings with central lines and dots surrounded by radiolucency[23]. But Chest CT is not an effective diagnostic tool for PIE presenting as multiple cysts with various sized in one or more lobes of the lung[14]. Especially, CT showed cystic lung lesions mimicking CCAM[6]. When a patient with the absence of classical CT features, PIE should be differentiated from other cystic lung lesions, including CCAM, CLE, lymphangiectasia, a bronchogenic cyst, cystic lymphangioma, and diaphragmatic hernia. According to the CT findings and clinical features, CCAM was suspected in $66.7 \%(4 / 6)$ cases in present study. Besides, there was only one case that prenatal ultrasound found cystic lesions in the previous literature[24]. Messineo et al. reported a male infant suffered from type I CCAM at 20 weeks of gestation with ultrasound scanning, which was diagnosed with PIE after surgery[24]. The prenatal ultrasound did not find any lesions in the lung of six patients with prenatal ultrasound examination in present study, which indicates that the PPIE may be formed after birth.

Persistent PIE is pathologically characterized by irregular-shaped and multiloculated cysts of various sizes along the bronchovascular bundle. And the cysts are air-filled spaces in the parenchymal and composed of a thin band of fibrous connective tissue. The degree of the fibrosis may vary the duration of PIE. The uninucleated and/or multinucleated macrophages lining the cyst walls are the typical pathological features[7, 8]. The typical features of air cysts surrounding the bronchovascular bundles with fibrous tissue lining the uninucleated and/or multinucleated macrophages were both demonstrated among all 6 cases in our present study.

The differential pathologically diagnosis was made with other cystic lesions such as CCAM, PS, bronchogenic cyst, CLE, and cystic lymphangioma and so on. CCAM is characterized by a lack of communication between the lesion and the tracheobronchial tree and a proliferation of irregularly dilated terminal bronchiole-like structures[25]. PS is characterized with non-functioning lung tissue receives systemic arterial blood supply and does not communicate with the adjacent tracheobronchial tree[26]. Bronchogenic cyst is lined with pseudostratified columnar respiratory epithelium, cartilage plate, smooth muscle, and bronchial glands[27]. CLE showed lobar hyperinflation with overdistention of normally formed alveoli and without destruction of alveolar walls, aspiration pneumonia, infection, and proximal bronchial obstruction[28]. 
Standard treatment strategy for PIE has not yet been established. Surgical resection of PIE is controversial, and several studies have advocated a conservative medical approach[23, 29]. When it is difficulty in making the diagnosis, the absence of classical CT features, and nonsurgical options failed or progressive syndrome, or severe complications, surgery should be considered[30]. Jassal et al. reported a case illustrates that extensive bilateral PPIE associated with a persistent pneumomediastinum can resolve spontaneously thus demonstrating that conservative management without surgical intervention may be appropriate for some children[29]. Infants with PPIE and weighing less than $1000 \mathrm{~g}$ are at significant risk of mortality and associated morbidity of PPIE[31].

The mechanism of production of PIE is the disruption of the alveolar wall basement membrane with subsequent dissection of air into the interstitial space. Given et al. [32] showed that PIE in bronchiolitis is thought to occur secondary to inflammation leading to mucosal edema, increased secretion and cellular debris, resulting in expiratory obstruction of the small airways, the resulting check-valve effect leads to hyperinflation then alveolar rupture. Another study[11] has demonstrated that pneumonia may contribute to the development of pulmonary air leaks by at least three mechanisms: firstly, air trapping from mechanical or check-valve obstruction within the bronchi by mucus and inflammatory exudates; Secondly, reduced strength or direct disruption of the alveolar lining from parenchymal inflammation or necrosis as commonly seen in necrotizing pneumonia, and thirdly, decreasing lung compliance.

There was obvious inflammatory reaction in present six rare cases. There are few reports of pulmonary air leakage with respiratory infection. There were just nine previous articles including 12 patients with PPIE with respiratory infection from a PubMed search[11-19]. Review of published literature of PIE with respiratory infection was showed inTable 3. Gala et al. [16] did not mention the sex of the case, so there were seven females and four males, ranged from birth to 87 years. Only $50 \%(6 / 12)$ patients with PPIE were treated with surgery. $72.2 \%(8 / 11)$ were full-term, and $58.3 \%(7 / 12)$ without mechanical ventilation. There was pulmonary air leakage, pneumothorax (5/12 cases) and pneumomediastinum (2/12 cases). According to the previous reported PIE patients with respiratory infection, there were nine patients without certain infectious pathogens reported[11,12,14,16-18], and the other three patients were infected by respiratory syncytial virus[13], Candida albicans[19], and respiratory sincitial virus[15], respectively. In our study, the common symptoms of the patients were cough, fever and expectoration. Special stains (acid fast stain, Gomori's methenamine silver staining, and Giemsa) and TB-PCR did not find any identifiable organism or foreign material in our study. Streptococcus pneumonia was detected in patient No.1, whose infectious symptoms were present when surgery. Neisseria mucosa, Neisseria sicca, Prevotel lamelaninogenica, Prevotella histicola, and Fusobacterium nucleatum were detected in patient No.5, and all the bacteria were Gram-negative. Patient No.5 has suffered the disease Langerhans cell histiocytosis (LCH) in the left submandibular lymph node for 1 year, and has received the suggested chemotherapy treatment four times, and then she had suffered recurrent pneumonia for three months, so the detected infectious pathogens may be associated with the chemotherapy treatment for LCH. No infectious pathogen was detected in other $66.7 \%(4 / 6)$ cases with pneumonia prior to surgery, whose infectious symptoms were controlled with conventional anti-infective treatment before surgery.

\section{Conclusion}

Six rare cases of PPIE with respiratory infection were successfully treated by lobectomy. All the available five cases were full-term infants without mechanical ventilation. The diagnoses of PPIE are based on characteristic radiographic and histologic findings. Moreover, no certain infectious pathogen found may be associated with PIE in children in our study.

\section{Abbreviations}

PIE: Pulmonary interstitial emphysema; PPIE: Persistent pulmonary interstitial emphysema; CT: Chest computed tomography; mNGS: metagenomic next-generation sequencing; CCAM: congenital cystic adenomatoid malformation; PS: pulmonary sequestration; CLE: congenital lobar emphysema;

\section{References}


1. Stocker LJ, Wellesley DG, Stanton MP, Parasuraman R, Howe DT: The increasing incidence of foetal echogenic congenital lung malformations: an observational study. Prenat Diagn 2015, 35(2):148153.https://doi.org/10.1002/pd.4507

2. Durell J, Lakhoo K: Congenital cystic lesions of the lung. Early human development 2014, 90(12):935939.https://doi.org/10.1016/j.earlhumdev.2014.09.014

3. Berk DR, Varich LJ: Localized persistent pulmonary interstitial emphysema in a preterm infant in the absence of mechanical ventilation. Pediatr Radiol 2005, 35(12):1243-1245.https://doi.org/10.1007/s00247$005-1562-\mathrm{z}$

4. Case records of the Massachusetts General Hospital. Weekly clinicopathological exercises. Case 301997. A preterm newborn female triplet with diffuse cystic changes in the left lung. N Engl J Med 1997, 337(13):916-924.https://doi.org/10.1056/NEJM199709253371308

5. Matta R, Matta J, Hage P, Nassif Y, Mansour N, Diab N: Diffuse persistent interstitial pulmonary emphysema treated by lobectomy. Ann Thorac Surg 2011, 92(4):e7375.https://doi.org/10.1016/j.athoracsur.2011.04.071

6. Aggarwal P, Mortellaro VE, St Peter SD: Pulmonary interstitial emphysema presenting as a congenital cystic adenomatous malformation on CT. Eur J Pediatr Surg 2011, 21(6):404-406.https://doi.org/10.1055/s0031-1279742

7. Sonnappa S, Cohen G, Ramsay A, Dinwiddie R, Jaffe A: A baby with cough and poor feeding. Localised persistent pulmonary interstitial emphysema of the left lung. Eur Respir J 2003, 22(1):182-185

8. Oh MH, Kim MY, Shim WS, Oh SS, Shin BK, Cho SJ, Kim HK: A case of localized persistent interstitial pulmonary emphysema. J Korean Med Sci 2001, 16(2):225-228.https://doi.org/10.3346/jkms.2001.16.2.225

9. Jones S, Kilgallon S, Schwartz AJ, Subramanyam R: Persistent Pulmonary Interstitial Emphysema. Anesthesiology 2018.https://doi.org/10.1097/ALN.0000000000002251

10. Hart SM, McNair M, Gamsu HR, Price JF: Pulmonary interstitial emphysema in very low birthweight infants. Arch Dis Child 1983, 58(8):612-615

11. Lee HS, Im SA: Pulmonary interstitial emphysema complicating pneumonia in an unventilated term infant. Indian J Pediatr 2010, 77(9):1025-1027.https://doi.org/10.1007/s12098-010-0154-6

12. Pursnani SK, Amodio JB, Guo H, Greco MA, Nadler EP: Localized persistent interstitial pulmonary emphysema presenting as a spontaneous tension pneumothorax in a full term infant. Pediatr Surg Int 2006, 22(7):613-616.https://doi.org/10.1007/s00383-006-1688-6

13. Aiyoshi T, Masumoto K, Shinkai T, Tanaka Y, Fujii S, Sasaki T, Chiba F, Sakamoto N, Gotoh C, Urita Y et al: Pulmonary interstitial emphysema due to respiratory syncytial virus infection. Pediatr Int 2016, 58(9):916-919.https://doi.org/10.1111/ped.13013

14. Cohen MC, Drut RM, Drut R: Solitary unilocular cyst of the lung with features of persistent interstitial pulmonary emphysema: report of four cases. Pediatr Dev Pathol 1999, 2(6):531-536

15. del Castillo BT, Gordillo I, Garcia ER, Lafever SNF, Cortes RG, Villaescusa JU, Gonzalez JL, Garcia MJS, Cid JLH: Diffuse persistent pulmonary interstitial emphysema secondary to mechanical ventilation in bronchiolitis. Bmc Pulmonary Medicine 2016, 16.https://doi.org/ARTN 139

10.1186/s12890-016-0299-9

16. Gala HC: Severe pulmonary interstitial emphysema in a premature baby. Indian J Pediatr 2014, 81(3):308-309.https://doi.org/10.1007/s12098-013-1264-8 
17. O'Donovan D, Wearden M, Adams J: Unilateral pulmonary interstitial emphysema following pneumonia in a preterm infant successfully treated with prolonged selective bronchial intubation. Am J Perinatol 1999, 16(7):327-331.https://doi.org/10.1055/s-2007-993880

18. Sherren PB, Jovaisa T: Pulmonary interstitial emphysema presenting in a woman on the intensive care unit: case report and review of literature. J Med Case Rep 2011, 5:236.https://doi.org/10.1186/1752-1947$5-236$

19. Yao JL, Fasano M, Morotti R, Caprio M, Greco MA: Demonstration of communication between alveolus and interstitium in persistent interstitial pulmonary emphysema: case report. Pediatr Dev Pathol 1999, $2(5): 484-487$

20. Gronbach J, Ehrhardt H, Zimmer KP, Waitz M: Early Pulmonary Interstitial Emphysema in Preterm Neonates-Respiratory Management and Case Report in Nonventilated Very Low Birth Weight Twins. AJP Rep 2018, 8(2):e99-e105.https://doi.org/10.1055/s-0038-1648253

21. Verma RP, Chandra S, Niwas R, Komaroff E: Risk factors and clinical outcomes of pulmonary interstitial emphysema in extremely low birth weight infants. J Perinatol 2006, 26(3):197200.https://doi.org/10.1038/sj.jp.7211456

22. Bawa P, Soontarapornchai K, Perenyi A, Goldfisher R, Amodio J: Development of Localized Pulmonary Interstitial Emphysema in a Late Preterm Infant without Mechanical Ventilation. Case Rep Pediatr 2014, 2014:429797.https://doi.org/10.1155/2014/429797

23. Donnelly LF, Lucaya J, Ozelame V, Frush DP, Strouse PJ, Sumner TE, Paltiel HJ: CT findings and temporal course of persistent pulmonary interstitial emphysema in neonates: a multiinstitutional study. AJR Am J Roentgenol 2003, 180(4):1129-1133.https://doi.org/10.2214/ajr.180.4.1801129

24. Messineo A, Fusaro F, Mognato G, Sabatti M, D'Amore ESG, Guglielmi M: Lung volume reduction surgery in lieu of pneumonectomy in an infant with severe unilateral pulmonary interstitial emphysema. Pediatr Pulm 2001, 31(5):389-393.https://doi.org/DOI 10.1002/ppul.1063.abs

25. Moerman P, Fryns JP, Vandenberghe K, Devlieger H, Lauweryns JM: Pathogenesis of congenital cystic adenomatoid malformation of the lung. Histopathology 1992, 21(4):315-321

26. Nakatani Y, Inayama Y, Kamijo S, Kawano N, Tanaka Y: Pathogenesis of pulmonary intralobar sequestration (ILS). Hum Pathol 1998, 29(9):1031-1032

27. McAdams HP, Kirejczyk WM, Rosado-de-Christenson ML, Matsumoto S: Bronchogenic cyst: imaging features with clinical and histopathologic correlation. Radiology 2000, 217(2):441446.https://doi.org/10.1148/radiology.217.2.r00nv19441

28. Mulvany JJ, Weatherall A, Charlton A, Selvadurai H: Congenital lobar emphysema: diagnostic and therapeutic challenges. BMJ Case Rep 2016, 2016.https://doi.org/10.1136/bcr-2016-214994

29. Jassal MS, Benson JE, Mogayzel PJ, Jr.: Spontaneous resolution of diffuse persistent pulmonary interstitial emphysema. Pediatr Pulmonol 2008, 43(6):615-619.https://doi.org/10.1002/ppul.20820

30. Rao J, Hochman MI, Miller GG: Localized persistent pulmonary interstitial emphysema. J Pediatr Surg 2006, 41(6):1191-1193.https://doi.org/10.1016/j.jpedsurg.2006.01.071

31. Yu VY, Wong PY, Bajuk B, Szymonowicz W: Pulmonary interstitial emphysema in infants less than $1000 \mathrm{~g}$ at birth. Aust Paediatr J 1986, 22(3):189-192

32. Given K, Schultz A, Douglas TA, Martin AC: Air leaks in children with acute bronchiolitis. J Paediatr Child Health 2008, 44(10):604-606.https://doi.org/10.1111/j.1440-1754.2008.01393.x 

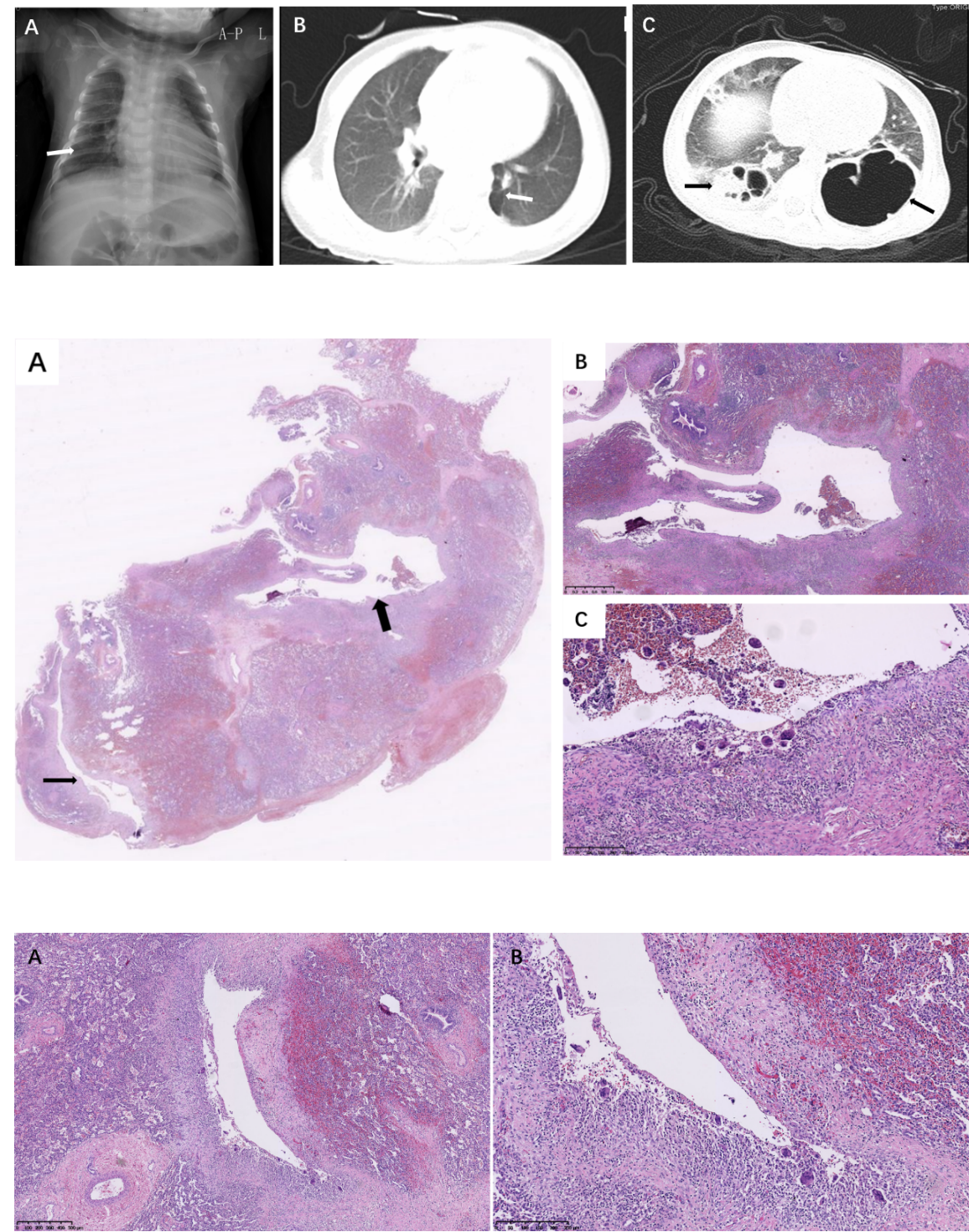

\section{Hosted file}

Tables.pdf available at https://authorea.com/users/368400/articles/487543-persistentpulmonary-interstitial-emphysema-with-respiratory-infection-a-clinicopathologicalanalysis-of-six-cases-and-detection-of-infectious-pathogens-by-metagenomic-nextgeneration-sequencing-mngs 